\title{
A New Model-CELBF for Medical Image Segmentation Based on Image Entropy
}

\author{
Chao Zhang ${ }^{1}$, Yu-Cui Guo², Feng-Shan Liu ${ }^{3}$ \\ ${ }^{1}$ Department of Mathematical Sciences Delaware State University, Dover, DE, USA \\ ${ }^{2}$ School of Science Beijing University of Posts and Telecommunications, Beijing, China \\ ${ }^{3}$ Department of Mathematical Sciences Delaware State University, Dover, DE, USA \\ ${ }^{1}$ czhang11@students.desu.edu, ${ }^{2} y c g u o @ b u p t . e d u . c n,{ }^{3} f l i u @ d e s u . e d u$
}

\begin{abstract}
A new model (named CELBF) for medical image segmentation based on LBF and image entropy is proposed in this paper. We introduced image entropy to deal with the inhomogeneity of image gray level. Some real medical images are processed by using this new model and finite difference algorithm. The results show that new model improves the speed of segmentation and increases noise robustness. Compared with LBF model, the new model can segment inhomogeneity medical image more quickly and more accurately. Meanwhile the CELBF model has more strong robustness with noise.
\end{abstract}

\section{Introduction}

Medical image is widely used in medical diagnosis. At present, the image segmentation methods are mostly used to locate the lesion boundary. The earlier image segmentation methods were based on low-level image knowledge, such as image gray, edge, and so on. Typical segmentation methods include threshold method, region growing method, edge detection method, etc. With the increasing of image types and higher quality demand, more and more high-level techniques are used for image segmentation, and therefore some classic methods were developed to solve the problems of complex image segmentation based on clustering, partial differential equations, and graph theory, etc. Among the classic methods, the image segmentation method based on partial differential equation (PDE) is one of the most popular methods in the field of image segmentation. A lot of good results have been developed based on image segmentation method and the image segmentation method is still widely used [1-5]. Partial differential equation (PDE) image segmentation is a nonlinear image segmentation method which was developed in 1980s. Its basic idea is to make the continuous mathematical model of the image to follow with a partial differential equation, and the corresponding segmentation result is obtained when the stable solution of the equation is obtained. The most important advantage of this technique is that the curve is continuous and smooth in the process of evolution. The classical model of image segmentation based on partial differential equation (PDE) is the active contour model which is based on the deformable model. The basic principle of the deformable model is that the curve moves and changes under the action of the driving forces including the internal and the external forces, in which the internal force is based on the geometric characteristics of the curve and keeps the smoothness of the curve; external force drives curve approximation to the boundary of the object through prior information of the target and the image information. Under the action of the internal force and the external force, the curve moves to the boundary of the target under the smoothness condition. The active contour model makes deformation of the curve through minimizing the energy. In the active contour model, an energy function of the curve is defined firstly, and then the given energy function is minimized to make the curve evolving to the boundary of the target.

In this paper, a new algorithm (named CELBF) for medical image segmentation based on information entropy is proposed. We combined the $\mathrm{CV}$ and the LBF models and used the concept of information entropy. The numerical results with MATLAB are satisfactory.

\section{Mathematical Basis of the Model}

Mathematics plays an important role in the development of image segmentation and penetrates into the various branches of image segmentation. PDE, as an applied mathematics, has become a hot topic in the field. Meanwhile, because the partial differential equation method is suitable for many kinds of images, it has very important theory and application value. The ideas of partial differential equations being applied to image processing can be traced back to the work of Gabor [6] 
in 1965 and Jain [7] in 1977. But a substantial progress thanks to Koenderink [8] and Witkin [9], for they put forward an idea of that signals and different scales of the convolution of Gauss function is equivalent to the heat diffusion equation with initial value of signal in 1984 and 1985 respectively, which was based on the theory of multi-scale analysis. Koenderink and Witkin also solved image denoising problems using the thermal diffusion equation. In 1993, an axiomatic system was formed by L. Alvarez [10] et al through the ingenious processing for the derivation of the Affine Morphological Scale Space equation, which marks that the formal formation of the discipline of image processing based on the partial differential equations.

During the last few decades, the image segmentation method based on differential equations has made great progress. Many kinds of typical active contour models based on geometric region have been proposed. Here we mainly introduce the CV model and the LBF model, because our new model in this paper is obtained mainly by introducing the image entropy method and by combining the CV model and the LBF model.

\subsection{Chan-Vese (CV) Model}

The CV model is a classical geometric active contour model based on region, which was proposed by Chan and Vese [3] in 2001. The CV model can be defined as that the image set $I$ defined in $\Omega$ is divided into two parts by the active contour $C$, and the two parts were recorded as inside $(C)$ and outside $(C)$. We use $c_{1}$ and $c_{2}$ to represent the average value of the internal gray level of the curve and the average value of the external gray level of the curve respectively. The fitting energy functional is defined as follows:

$$
\begin{aligned}
E_{C V}\left(C, c_{1}, c_{2}\right)= & \lambda_{1} \iint_{\text {inside }(C)}\left|I(x, y)-c_{1}\right|^{2} d x d y \\
& +\lambda_{2} \iint_{\text {outside }(C)}\left|I(x, y)-c_{2}\right|^{2} d x d y \\
& +u \operatorname{uLngth}(C)+\operatorname{virea}(\operatorname{inside}(C))
\end{aligned}
$$

where Length $(C)$ is the length of the contour curve which plays a role of regularization, Area(inside $(C)$ ) is the internal area of the contour curve, $u, v \geq 0$ are two parameters; $\lambda_{1}, \lambda_{2}$ are two weight coefficients, and $\lambda_{1}, \lambda_{2}>0$. The first two terms on the right hand side of the formula are called the "fitting" terms which are mainly used to control the fitting error for the contour curve fit edge. The other two terms are called the "smooth" terms for getting a smooth curve in the process of evolution. The final position of the contour curve can be obtained by minimizing the energy functional.

Let the level set function $\phi$ be represented by the following formula:

$$
\begin{cases}\phi(x, y)=0, & (x, y) \in C \\ \phi(x, y)>0, & (x, y) \in \text { inside }(C) \\ \phi(x, y)<0, & (x, y) \in \text { outside }(C) .\end{cases}
$$

In order to apply the level set method, the Heaviside function is introduced

$$
H(x)= \begin{cases}1, & x \geq 0 \\ 0, & x<0\end{cases}
$$

And the Dirac function is as

$$
\delta(x)=\frac{d}{d x} H(x)
$$

In this method, the Heaviside function is used to divide the region of evolution, and the Dirac function is used to define the value of evolution around the zero level set function. Here,

$$
\begin{gathered}
\text { Length }(C)=\int_{0}^{L(C)} d s=\iint_{\Omega} \delta(\phi)|\nabla \phi| d x d y, \\
\text { Area(inside }(C))=\iint_{\Omega} H(\phi) d x d y, \\
\iint_{\text {inside }(c)}\left|I(x, y)-c_{1}\right|^{2} d x d y \\
=\iint_{\Omega}\left|I(x, y)-c_{1}\right|^{2} H(\phi) d x d y, \\
\iint_{\text {outside }(c)}\left|I(x, y)-c_{2}\right|^{2} d x d y \\
=\iint_{\Omega}\left|I(x, y)-c_{2}\right|^{2}(1 \\
-H(\phi)) d x d y .
\end{gathered}
$$

Thus, the energy functional of the level set function is obtained as

$$
\begin{aligned}
E_{C V}\left(\phi, c_{1}, c_{2}\right) & =\lambda_{1} \iint_{\Omega}\left|I(x, y)-c_{1}\right|^{2} H(\phi) d x d y \\
& +\lambda_{2} \iint_{\Omega}\left|I(x, y)-c_{2}\right|^{2}(1 \\
& -H(\phi)) d x d y \\
& +u \iint_{\Omega} \delta(\phi)|\nabla \phi| d x d y \\
& +v \iint_{\Omega} H(\phi) d x d y .
\end{aligned}
$$

At this point, the level set of the image $I(x, y)$ is:

$$
I(x, y)=c_{1} H(\phi)+C_{2}(1-H(\phi)) .
$$

Fixing $\phi$ and minimizing the energy functional $E_{C V}\left(\phi, c_{1}, c_{2}\right)$, we have 


$$
\begin{gathered}
c_{1}=\frac{\iint_{\Omega} I(x, y) H(\phi) d x d y}{\iint_{\Omega} H(\phi) d x d y}, \\
c_{2}=\frac{\iint_{\Omega} I(x, y)(1-H(\phi)) d x d y}{\iint_{\Omega}(1-H(\phi)) d x d y} .
\end{gathered}
$$

In the CV model, usually we set $v=0$. The energy functional $E_{C V}\left(\phi, c_{1}, c_{2}\right)$, is minimized using the variation method and gradient descent flow technique and Euler-Lagrange equation is obtained as

$$
\begin{gathered}
\frac{\partial E}{\partial \phi}=-\delta(\phi)\left(u \cdot \operatorname{div}\left(\frac{\nabla \phi}{|\nabla \phi|}\right)-\lambda_{1}\left(I-c_{1}\right)^{2}+\lambda_{2}\left(I-c_{2}\right)^{2}\right) \\
=0 .
\end{gathered}
$$

The regularized Heaviside function is often used in the numerical calculation as

$$
H_{\varepsilon}(x)=\frac{1}{2}\left[1+\frac{1}{\pi} \arctan \left(\frac{x}{\varepsilon}\right)\right]
$$

and the corresponding Dirac function is

$$
\delta(x)=\frac{1}{\pi} \frac{\varepsilon}{\varepsilon^{2}+x^{2}} .
$$

Our goal is to expand the capture range and to prevent the occurrence of singular cases. For the $\mathrm{CV}$ model, due to the fact that both $c_{1}$ and $c_{2}$ represent the average gray values of the internal and external of the contour curve and therefore are global variables, CV model is a segmentation model based on global information. Although it has advantages with respect to noise robustness, calculation simplicity, etc., it cannot be used for non-uniform gray image segmentation.

\subsection{Local Binary Fitting (LBF) Model[3,4,11]}

Let $x$ be a point in the image area and $C$ be the contour curve, and the fitting energy function at the point $x$ is defined as

$$
\begin{gathered}
E_{x}^{\text {Fit }}=\lambda_{1} \int_{\text {inside }(C)} K_{\sigma}(x-y)\left|I(y)-f_{1}(x)\right|^{2} d y \\
\quad+\lambda_{2} \int_{\text {outide }(C)} K_{\sigma}(x \\
-y)\left|I(y)-f_{2}(x)\right|^{2} d y
\end{gathered}
$$

where $f_{1}(x)$ and $f_{2}(x)$ are gray fitting values of the image at the point of $x, K_{\sigma}$ is the Gauss kernel function with a standard deviation of $\sigma$, and

$$
K_{\sigma}=\frac{1}{\sqrt{2 \pi} \sigma} e^{-\frac{|x|^{2}}{2 \sigma^{2}}} .
$$

Let $\phi$ be the level set function of contour curve C. Using the level set method, we have

$$
\begin{gathered}
E_{x}^{\text {Fit }}=\lambda_{1} \int_{\text {inside }(C)} K_{\sigma}(x-y)\left|I(y)-f_{1}(x)\right|^{2} H(\phi) d y \\
+ \\
\lambda_{2} \int_{\text {outide }(C)} K_{\sigma}(x-y)\left|I(y)-f_{2}(x)\right|^{2}(1-H(\phi)) d y .
\end{gathered}
$$

The total fitting energy function is

$$
E^{F i t}=\int_{\Omega} E_{x}^{F i t} d x
$$

Adding the set regularization $\int_{\Omega} \frac{1}{2}(|\nabla \phi|-1)^{2} d \sigma$ term, the total energy functional of LBF model is

$$
E^{L B F}=E^{F i t}+\int_{\Omega} \frac{1}{2}(|\nabla \phi|-1)^{2} d \sigma+v|C| .
$$

Firstly fixing $\phi$ in above formula, after minimizing the energy functional $E^{L B F}$ with respect to $f_{1}(x)$ and $f_{2}(x)$, we have

$$
\begin{gathered}
f_{1}(x)=\frac{\int_{\Omega} K_{\sigma}(x-y) I(y) H(\phi(y)) d y}{\int_{\Omega} K_{\sigma}(x-y) H(\phi(y)) d y} \\
f_{2}(x)=\frac{\int_{\Omega} K_{\sigma}(x-y) I(y)(1-H(\phi(y))) d y}{\int_{\Omega} K_{\sigma}(x-y)(1-H(\phi(y))) d y} .
\end{gathered}
$$

Then fixing the fitting factors $f_{1}(x)$ and $f_{2}(x)$, after minimizing the energy functional $E^{L B F}$ with respect to $\phi$, the evolution equation of the energy functional $E^{L B F}$ is obtained using a gradient descent flow:

$$
\begin{gathered}
\frac{\partial \phi}{\partial t} \\
=-\delta_{\varepsilon}(\phi)\left(\lambda_{1} e_{1}-\lambda_{2} e_{2}\right)+u\left[\Delta \phi-\operatorname{div}\left(\frac{\nabla \phi}{|\nabla \phi|}\right)\right] \\
+v \delta_{\varepsilon}(\phi) \operatorname{div}\left(\frac{\nabla \phi}{|\nabla \phi|}\right)
\end{gathered}
$$

where

$$
\begin{aligned}
& e_{1}=\int_{\Omega} K_{\sigma}(x-y)\left|I(x)-f_{1}(y)\right|^{2} d y, \\
& e_{2}=\int_{\Omega} K_{\sigma}(x-y)\left|I(x)-f_{1}(y)\right|^{2} d y .
\end{aligned}
$$

In the LBF model, $f_{1}(x)$ and $f_{2}(x)$ are the fitting energy of the image at the point $x$ which are local energy related variance. Therefore, the LBF model is based on the local image information. Meanwhile, due to the introduction of the local energy, the LBF model has good segmentation effect for inhomogeneity image. Its disadvantages are that it is sensitive for contour initialization and poor noise robustness, etc. 


\section{A New Model Celbf Based On Information Entropy}

Considering the advantages and the disadvantages of the CV model and the LBF model [12], we created a new local fitting energy functional to replace the ones in the $\mathrm{CV}$ and the LBF models. The new energy functional is defined as:

$$
\begin{aligned}
E^{N L G F}=\omega E^{G I F}+ & (1-\omega) E^{N L I F}+v L(\phi) \\
& +\mu P(\phi)
\end{aligned}
$$

Where

$$
\begin{aligned}
E^{G I F}=\lambda_{1} & \iint_{\text {inside }(C)}\left|I(x, y)-c_{1}\right|^{2} d x d y \\
& +\lambda_{2} \iint_{\text {outside }(C)}\left|I(x, y)-c_{2}\right|^{2} d x d y
\end{aligned}
$$

is a global fitting factor,

$$
E^{N L I F}=\sum_{i=1}^{2} \int_{\Omega} \lambda_{i}\left|I(x, y)-f_{i}(x)\right|^{2} M_{i}(\phi) d x
$$

is a new local fitting energy functional level set, and

$$
\begin{gathered}
f_{1}(x)=\frac{K(x) *(I(x) H(\phi))}{K(x) * H(\phi)}, \\
f_{2}(x)=\frac{K(x) *(I(x)(1-H(\phi)))}{K(x) *(1-H(\phi))} .
\end{gathered}
$$

Use gradient descent to solve the equation (3.1), we have the final curve evolution equation:

$$
\begin{gathered}
\frac{\partial \phi}{\partial t} \\
=-\delta_{\varepsilon}(\phi)\left(F_{1}+F_{2}\right)+u\left[\Delta \phi-\operatorname{div}\left(\frac{\nabla \phi}{|\nabla \phi|}\right)\right] \\
+v \delta_{\varepsilon}(\phi) \operatorname{div}\left(\frac{\nabla \phi}{|\nabla \phi|}\right)
\end{gathered}
$$

$$
\begin{aligned}
& \text { Where } F_{1}=\omega\left(\lambda_{1}\left(I(x)-c_{1}\right)^{2}-\lambda_{2}\left(I(x)-c_{2}\right)^{2}\right) \\
& \begin{aligned}
F_{2}=(1-\omega) & \left(\lambda_{1} \int_{\Omega} K_{\sigma}(y-x)\left|I(x)-f_{1}(y)\right|^{2} d y\right. \\
& -\lambda_{2} \int_{\Omega} K_{\sigma}(y \\
& \left.-x)\left|I(x)-f_{2}(y)\right|^{2} d y\right)
\end{aligned}
\end{aligned}
$$

We call $F_{1}$ and $F_{2}$ the global intensity fitting (GIF) force and the local intensity fitting (LIF) force, respectively.

In order to deal with the gray inhomogeneity of images, we introduce the concept of image entropy in our model. The image entropy was introduced for the first time to image segmentation in 1972 by Freden [13]. From then, the concept of entropy has played an important role. At present, image entropy is commonly used in image segmentation.

Let $p_{i}$ be a distribution of a given image $I_{i}$. Based on Shannon's information theory, the image entropy is

$$
E_{I}=-\sum_{i=1}^{n} p_{i} \log p_{i}
$$

Where the distribution function of the image $p_{i}$ can be given by a variety of ways depending on the segmentation method.

In this paper, let $I: \Omega \subset \mathrm{R}^{2} \rightarrow R$ be an image and $\Omega_{x} \subset \Omega$ be a neighborhood of $\mathrm{x}$ with a radius of $\mathrm{r}$. Then the local entropy for point $\mathrm{x}$ is defined as

$$
E\left(x, \Omega_{x}\right)=-\frac{1}{\log \left|\Omega_{x}\right|} \int_{\Omega_{x}} p\left(y, \Omega_{x}\right) \log p\left(y, \Omega_{x}\right) d y
$$

Where $p\left(y, \Omega_{x}\right)$ is the distribution function of gray level $[10,14]$ which is shown as

$p\left(y, \Omega_{x}\right)=\frac{I(y)}{\int_{\Omega_{x}} I(z) d z}, y \in \Omega_{x}$.

The reason for the LBF model being sensitive to noise is that the gray level variation of image is not considered when the local fitting energy $E^{L B F}$ is defined. In this paper, we consider the gray variation information and also modify the energy in (2.1). Then we propose a weighted LBF model that is based on image entropy to improve robustness to the noise. We define this model as CELBF model. We define a new fitting energy functional at contour curve $\mathrm{C}$ of $\mathrm{LBF}$ model as

$$
E\left(C, f_{1}, f_{2}\right)=\int_{\Omega} E_{r}(x) E_{x}\left(C, f_{1}(x), f_{2}(x)\right) d x
$$

Where $E_{r}(x)=E(x, B(x, r))$ is the local entropy for point $x$.

By minimizing the energy functional, we obtain the level set differential equation of our model

$$
\begin{gathered}
\frac{\partial \phi}{\partial t}=-\delta_{\varepsilon}(\phi) \\
\left(F_{1}+F_{2}\right)+u\left[\Delta \phi-\operatorname{div}\left(\frac{\nabla \phi}{|\nabla \phi|}\right)\right] \\
+v \delta_{\varepsilon}(\phi) \operatorname{div}\left(\frac{\nabla \phi}{|\nabla \phi|}\right)
\end{gathered}
$$

Where $F_{1}=\omega\left(\lambda_{1}\left(I(x)-c_{1}\right)^{2}-\lambda_{2}\left(I(x)-c_{2}\right)^{2}\right)$

$$
\begin{aligned}
F_{2}=(1-\omega)( & \lambda_{1} \int_{\Omega} E_{r}(x) K_{\sigma}(y-x)\left|I(x)-f_{1}(y)\right|^{2} d y \\
& -\lambda_{2} \int_{\Omega} E_{r}(x) K_{\sigma}(y \\
& \left.-x)\left|I(x)-f_{2}(y)\right|^{2} d y\right) .
\end{aligned}
$$


With this model, several groups of images were segmented by using finite difference method and MATLAB. Comparing with the results of LBF model, our results are satisfactory.

\section{Result and analysis}

Using our new CELBF model, we obtained better results than the $\mathrm{CV}$ model and the LBF model.

\subsection{Segmentation Time}

Figure 4.1 shows the segmentation results of two typical gray inhomogeneous images, which both are taken from the literature [13]. One is a true T-type image $(127 * 90)$, and the other is the X-ray blood vessel image $\left(111^{*} 110\right)$. The segmentation results of the two images are obtained from the CELBF model and the LBF model respectively using the same parameters of $\quad \lambda_{1}=\lambda_{2}=1, \mu=1, \Delta t=0.1, h=1, \sigma=3$ and $\omega=0.01$ and 0.065 for CELBF model. It is not difficult to see that the CELBF model achieves almost the same segmentation results as the LBF model from the figures. However, the CELBF model used fewer iterations and faster segmentation speed. The speed of the CELBF model is approximately $1 / 2$ of the LBF model. It is show that the CELBF model has greatly improved the speed of segmentation while ensuring the accuracy

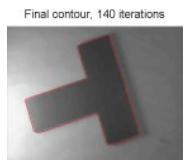

(a)

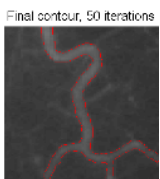

(c)

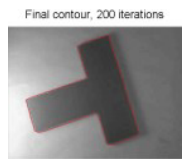

(b)

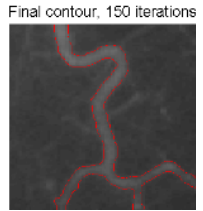

(d)
Figure 4.1

\subsection{Segmentation for Magnetic Resonance Imaging (MRI)}

Segmentation methods are widely used for dealing with MRI images. Figure 4.2 (a) $(189 * 164 * 3)$ and 4.2 (e) $(157 * 141)$ are two real human brain Magnetic Resonance Imaging. The segmentation results of the two images are obtained from the CELBF model and the LBF model with the same parameters of $\lambda_{1}=\lambda_{2}=$ $1, \mu=1, \Delta t=0.1, h=1, \sigma=3$, and $\omega=0.35$ in our CELBF model. Figure 4.2 (b, c, d) and Figure 4.2 (f, g, h) are segmentation results obtained by using the LBF model with 60, 200 and 500 iterations respectively. The segmentation results show that our CELBF model has better segmentation ability for complex brain MRI images. Our model ensures the accuracy of segmentation and improves segmentation speed. LBF model cannot get accurate segmentation results on complex images, and therefore it shows that the CELBF model works well with variance of grayscale level of the image to a certain extent.

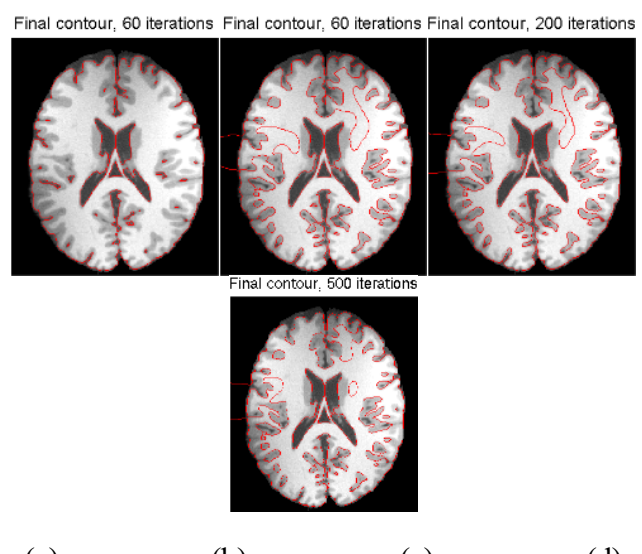

(a)

(b)

(c)

(d)
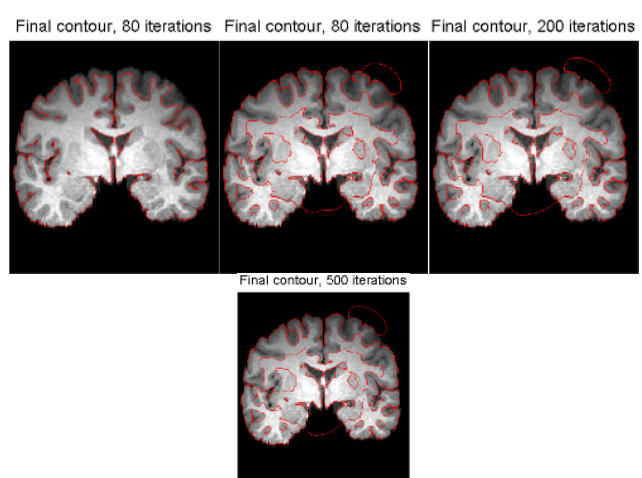

(d)

(e)

(f)

(g)

Figure 4.2

\subsection{Noise Sensitivity Degree}

Figure 4.3 (a) and 4.3 (c) are the segmentation results of human brain Magnetic Resonance Imaging (MRI) (119*78)using the CELBF model and the LBF model under the same parameters. It is easy to see that the results are same under both models without noise; furthermore the CELBF model is faster than LBF. Figure 4.3(b) and Figure 4.3(d) are segmentation results with Poisson noise using the two models. We can see from the results that noise has some effect for both models, but it has more effect for the LBF model.

Final contour, 200 iterations

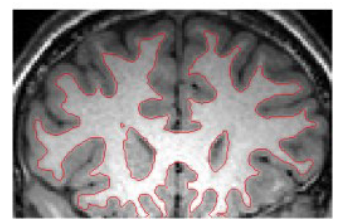

(a)
Final contour, 200 iterations

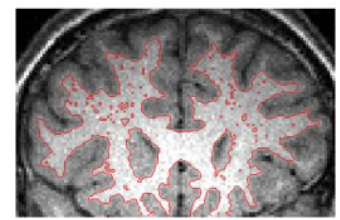

(b) 
Final contour, 300 iterations

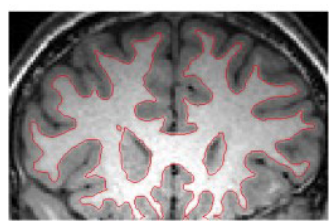

(c)
Final contour, 200 iterations

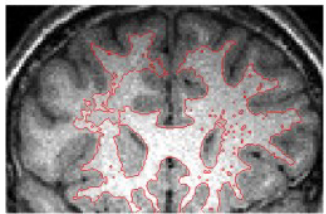

(d)
Figure 4.3

Figure 4.4 (b) and Figure 4.4(c) are segmentation results for a simple synthetic image $(64 * 61)$ using the two models. This image is relatively simple for segmentation because of the target and the background has obvious contrast. Therefore, both two models achieve good results. But CELBF model is faster than the LBF model. Figure 4.4(b) and 4.4(d) are segmentation results for the image with noise. Even though we have segmented some part of the target. It is obvious that CELBF model can obtained less unnecessary segment. It means that CELBF greatly reduces the sensitivity to noise.

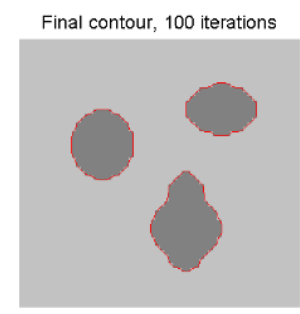

(a)

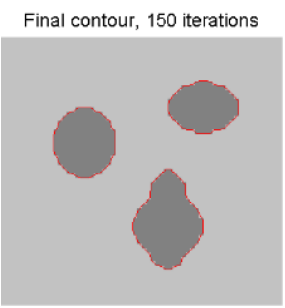

(c)

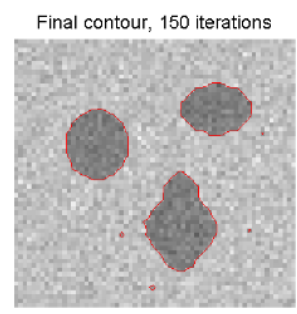

(b)

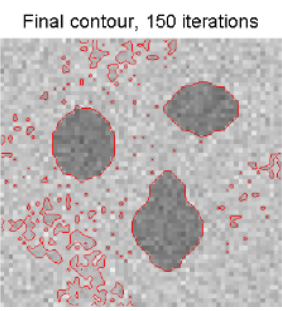

(d)
Figure 4.4

Figure 4.5 (a) shows the segmentation results of a human brain MRI $(189 * 163 * 3)$ for the CELBF model. Figure 4.5 (b) and 4.5 (c) are the results with Gaussian noise with standard deviations of 0.01 and 0.02 , respectively. All of these three images have been accurately segmented. It can be seen from the experimental results that the noise has a certain impact on our model, but the impact is minimal.

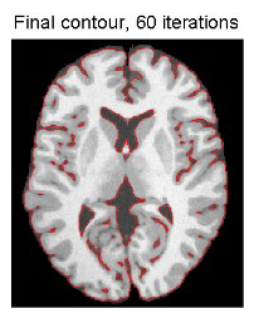

(a)

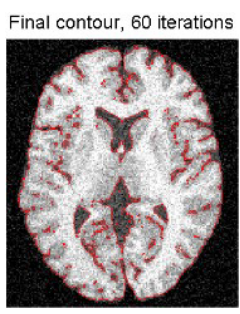

(b)

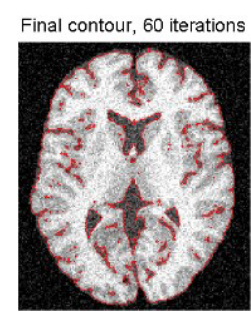

(c)
Figure 4.5

The experimental results illustrate further that the CELBF model has more advantages on medical images with gray inhomogeneity, weak edge images and complex background images. CELBF has strong robustness to noise, and so on.

\section{Conclusion}

Image segmentation methods based on partial differential equation is widely used. However, the existing models are generally sensitive to noise, and lack of robustness and evolution speed. In this paper, we propose an improved LBF model based on image entropy (defined as CELBF). Using this model and MATLAB, several groups of medical images segmentation are simulated. The segmentation results show that our CELBF model improves the robustness of the LBF model and the segmentation speed at the same time. Therefore, the CELBF model can better deal with medical images with gray inhomogeneity. Our model has advantages with respect to segmentation speed, accuracy and noise robustness.

\section{References}

[1] M. Kass, A. Witriw, and D. Terzopoilos, "Snakes: active contour models," International Journal of Computer Vision, 1988, 1:321-369

[2] V. Caselles, F. Catte, T. Coll, and F. Dibos, "A geometric model for active contours in image processing," Numerische Mathmatik., 1993,66:1-31.

[3] T. Chan, and L. Vese, "Active contours without edge," IEEE Transactions on Image Processing, 2001,10(2):266-277..

[4] C. Li, C. Kao, C. Gore, and Z. Ding, "Implicit active contours driven by local binary fitting energy," IEEE Computer Vision and Pattern Recognition,2007,6:17-22.

[5] C. Li, C. Xu, C. Gui, and M. D. Fox, "Level set evolution without re-initialization; a new variational formulation," IEEE Computer Society Conference on Computer Vision and Pattern Recognition, 2005, 1:430-436

[6] D. Gabor, "Information theory in electron microscopy," Laboratory Investigation, 1965, 14:801-807. 
[7] A. K. Jain, "Partial differential equations and finite-difference methods in image processing, part 1: Image representation," Optimization Theory and Applications, 1997, 23: 65-91.

[8] J. J. koenderink, "The structure of images," Biological Cybernetics, 1984, 50:363-370.

[9] A. P. Witkin, "Scale-space filtering," Proceedings of the 8th International Joint Conference Artificial Intelligence (IJCAI), Karlsruhe Germany, 1983, 2:1019-1021.

[10]L. Alvarez, F. Guichard, P. L. Lions, and J. M. Morel, et.al. "Axioms and fundamental euations of image processing," Archive for Rational Mechanics and Analysis, 1993, 16(9):200-257.

[11]C. Li, C. Kao, J. C. Gore, and Z. Ding, "Minimization of region-scalable fitting energy for image segmentation," IEEE Transactions on Image Processing, 2008, 17(10):1940-1949.

[12]L. Wang, C. Li, Q. Sun, D. Xia, and C. Kao, "Active contours driven by local and global intensity fitting energy with application to brain MR image segmentation," Computerized Medical Imaging and Graphics, 2009, 33:520-531.

[13]B. R. Frieden, "Restoring with maximum likelihood and maximum entropy," Journal of the Optical Society of America, 1982, 62(4):511-518.

[14]M. Sezgin, and B. Sankur, "Survey over image thresholding techniques and quantitative performance evaluation," Journal of Electronic Imaging, 2004, 13(1):146-165. 